\title{
Femtosecond two-photon ionization spectroscopy of the B state of $\mathrm{Na}_{3}$ clusters
}

\author{
T. Baumert, R. Thalweiser and G. Gerber \\ Fakultät für Physik, Universität Freiburg, W-7800 Freiburg, Germany
}

Received 6 April 1993

\begin{abstract}
We report time-resolved experiments studying the dynamics of the $\mathrm{Na}_{3} \mathrm{~B}-\mathrm{X}$ system. Femtosecond pump-probe techniques combined with ion time-of-flight (TOF) and zero kinetic energy (ZEKE) photoelectron spectroscopy allow us to observe the three-dimensional wavepacket motion in the excited $\mathrm{Na}_{3} \mathrm{~B}$ state and in the $\mathrm{Na}_{3} \mathrm{X}$ state. The ground state wavepacket is induced by stimulated emission pumping during the pump pulse. The $\mathrm{X}$-state dynamics is dominated by the three vibrational modes of the $\mathrm{Na}_{3}$. Furthermore we observed pseudorotational wavepacket motion in the B state. We do not observe a fragmentation of the $B$ state within a time interval of $10 \mathrm{ps.}$
\end{abstract}

\section{Introduction}

In recent years metal clusters have been the subject of many experimental and theoretical publications. Cluster physics form the link between molecular and solid state physics. Cluster-size-dependent studies of physical properties such as geometric structure, ionization potential and dissociation energy, or the size-dependent evolution of localized electronic excitations in small metal clusters to collective plasmon-like excitations of larger systems yield important information about the transition from molecular to solid-state-like behaviour. Sodium clusters are the theoretically and experimentally most studied metal clusters. They can be produced easily in "seeded beam" expansions and they can be excited or ionized by convenient available laser sources. Since there is only one valence electron per atom, sodium clusters are very attractive and facilitate theoretical studies.

The $\mathrm{Na}_{3}$ cluster has already been investigated extensively (see for example ref. [1]). Very recently we determined the geometrical structures of the neutral clusters $\mathrm{Na}_{3}$ and $\mathrm{Na}_{4}$ and of their positive ions by ZEKE photoelectron spectroscopy [2]. In earlier work we reported on experiments applying ion and photoelectron TOF spectroscopy to study the fragmentation processes in two-photon ionization of $\mathrm{Na}_{3}$ via the D state [3]. The electronic excitations of $\mathrm{Na}_{3}$ have been investigated experimentally by resonant two-photon ionization (TPI) spectroscopy [4] and by depletion spectroscopy [5]. In the case of larger sodium clusters the TPI spectroscopy with nanosecond lasers fails due to the very short lifetimes (0.33$3.5 \mathrm{ps}$ ) of the excited electronic states [6]. Therefore techniques such as depletion spectroscopy or femtosecond resonance enhanced multiphoton ionization (REMPI) [7] are suitable for studies of the absorption resonances. Many different theoretical methods [8] from the simple Hückel model to complex calculations on full $\mathrm{CI}$ level have been applied to investigate geometrical structures, electronic excitations and ionization potentials of sodium clusters. Cocchini et al. [9] calculated the excited electronic states of $\mathrm{Na}_{3}$ up to an energy of $2.7 \mathrm{eV}$. These calculations show that most excited electronic states are distorted by the Jahn-Teller effect. The $\mathrm{Na}_{3} \mathrm{X}$ state and the $\mathrm{B}$ state also fall into this category. Hermann et al. [10] reported on the first observation of the $\mathrm{Na}_{3} \mathrm{~B}$ state. Later Delacretaz et al. [11] reinvestigated the $\mathrm{Na}_{3} \mathrm{~B}$ state by resonant two-photon ionization using nanosecond laser pulses. They originally interpreted their high-resolution measurements in the framework of a pure Jahn-Teller model and half-integer $j$ values. In contrast to this interpretation the calculations of Meiswinkel and Köppel 
[12] showed that the TPI spectrum of Delacretaz et al. could alternatively be well understood by applying a pseudo-Jahn-Teller model and integer $j$ values. Recently Ernst et al. [13] have reinvestigated the B$\mathrm{X}$ system with rotational resolution by using $\mathrm{cw}$ resonant two-photon ionization spectroscopy. These authors believe their rotationally resolved spectra can only be explained in terms of integer quantum numbers $j$ of the vibronic angular momentum.

The first lifetime measurements of electronically excited states of $\mathrm{Na}_{3}$ have been reported by Broyer et al. [14]. They have used nanosecond time-delayed two-photon ionization techniques and found for the decay time of the $\mathrm{Na}_{3} \mathrm{~B}$ state a value of $\tau=14$ ns. More recently Wöste and co-workers [15] have reported time-resolved two-photon ionization spectroscopy of the $\mathrm{B}$ state of $\mathrm{Na}_{3}$ employing picosecond pump-probe techniques. Their main result obtained with 1.25 ps light pulses tunable in the range $600-$ $633 \mathrm{~nm}$ with a bandwidth of about $15 \mathrm{~cm}^{-1}$ is the observation of a fast decay with a 6 ps time constant (in ref. [16] they give 3.1-4 ps) superimposed by an oscillation with a time period of approximately 3 ps. The oscillating signal is interpreted as a pseudorotational motion in the $\mathrm{B}$ state.

Femtosecond pump-probe studies in the gas phase, which have been pioneered by Zewail and co-workers [17], have shown that molecular motions can now be probed in real-time. The time evolution of the induced coherences have been investigated by measuring selected fluorescence from excited states [17] or by ionizing the molecule and measuring transient ionization spectra [18].

In our studies we used femtosecond pump-probe techniques to investigate the real-time dynamics of two-photon ionization and fragmentation of the $\mathrm{Na}_{3}$ $\mathrm{B}-\mathrm{X}$ system. With a tunable pump laser photon a number of vibrational states of the B state are coherently excited and a time delayed identical probe laser is used to ionize the $\mathrm{Na}_{3}^{*}$ and to monitor the remaining excited state population. The formed cluster ions $\left(\mathrm{Na}_{3}^{+}\right)$are mass selected by a time-offlight (TOF) mass spectrometer. In addition to the detection of the $\mathrm{Na}_{3}^{+}$ions, the ZEKE (zero kinetic energy) electron signal has also been measured as a function of the delay time between the pump and probe pulse.

\section{Experiment}

The sodium clusters $\mathrm{Na}_{3}$ are produced in a "seeded beam" expansion. Sodium vapor (50-100 mbar) is coexpanded with the inert carrier gas argon ( $1-8$ bar) through a small cylindrical nozzle of $100 \mu \mathrm{m} \mathrm{di}$ ameter. The vibrational temperature of the $\mathrm{Na}_{3}$ clusters is about $40 \mathrm{~K}$ as inferred from vibrational ZEKE spectroscopy [2].

The laser system and the schematic experimental arrangement of the cluster beam and the TOF spectrometers are shown in fig. 1. Femtosecond pulses are generated in a home-built CPM (colliding pulse mode-locked) ring dye laser. The output of the CPM was amplified at a rate of $100 \mathrm{~Hz}$ in a multipass dye amplifier and/or in a two-stage dye amplifier, both pumped by a $308 \mathrm{~nm}$ excimer laser synchronized to

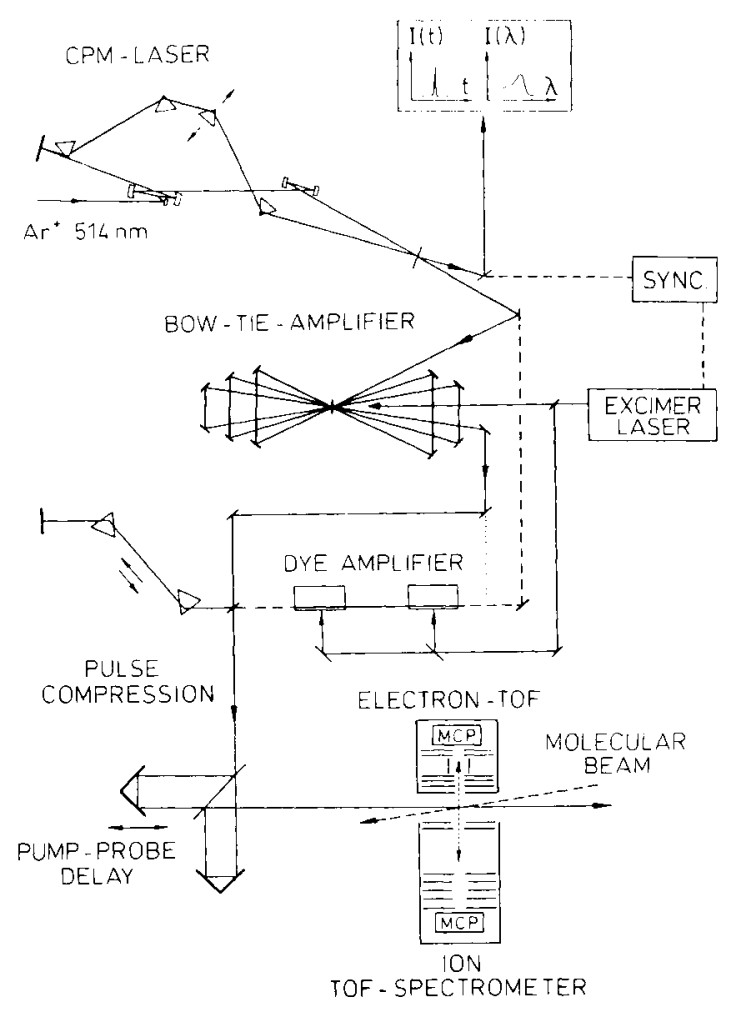

Fig. 1. Femtosecond pump-probe laser system and cluster beam arrangement. Femtosecond pulses ( $\tau=60 \mathrm{fs}$ ) are generated by a colliding-pulse mode-locked (CPM) ring dye laser, amplified in dye amplifiers, pulse compressed, and split into identical pump and probe pulses. Pump and probe laser beams are collinear and perpendicular to the cluster beam and the TOF spectrometers. 
the CPM. For the experiments described in this paper we employed recompressed ultrashort laser pulses of $60 \mathrm{fs}\left(\mathrm{sech}^{2}\right)$ time duration with nearly bandwidth limited spectral distributions of about 250 $\mathrm{cm}^{-1}$ and of $1 \mu \mathrm{J}$ energy. The laser pulse energy was kept this low in the pump-probe experiments to study the basic physical processes. The central wavelengths of 618 and $623 \mathrm{~nm}$ have been obtained by adjusting the concentrations of the gain (Rh6G) and the $a b$ sorber (DODCI) medium of the CPM dye laser. For the pump-probe experiments we used a Michelson arrangement to delay the probe laser relative to the pump laser. Both the pump and the probe laser beams enter the interaction region collinearly with the same polarization and perpendicular to the cluster beam.

The pump laser forms a coherent superposition of a number of vibrational and pseudorotational levels, i.e. it generates a wavepacket motion on the B-state potential surface. The time-delayed probe laser monitors this motion by photoionizing the excited $\mathrm{Na}_{3}^{*}$ clusters. The cluster ions $\mathrm{Na}_{3}^{*}$ are detected in a TOF mass spectrometer. In addition to the $\mathrm{Na}_{3}^{+}$ions produced by resonant two-photon ionization via the $B$ state we measured the corresponding ZEKE photoelectron signal as a function of the time delay between the identical pump and probe pulses. For those experiments we used a specially designed ZEKE photoelectron spectrometer.

The technique of ZEKE electron spectroscopy, originally proposed by Müller-Dethlefs and Schlag [19], briefly consists of the following. The clusters are photoionized by a short laser pulse in a field-free region under spatially and temporally well defined conditions. After an adjustable time delay (1-5 $\mu \mathrm{s})$ a small pulsed electric field is applied to extract the photoelectrons. During a time delay of $1 \mu$ s kinetic photoelectrons will move away and experience a different potential at the application of the electric field than the resting ZEKE electrons. This leads to different flight times and therefore the ZEKE photoelectrons can be separated by gated detection techniques. Besides that temporal effect a ZEKE photoelectron spectrometer has a very narrow acceptance angle leading to an additional steradiancy discrimination. The energy resolution of our ZEKE spectrometer is about $1 \mathrm{~cm}^{-1}$.

\section{Results and discussion}

In an earlier publication we reported on femtosecond wavepacket dynamics in multiphoton ionization and fragmentation of $\mathrm{Na}_{2}$ [18]. Compared to that experiment the dynamics in the two-photon ionization process of $\mathrm{Na}_{3}$ via the resonant $\mathrm{B}$ state is much more complex. Fig. 2a shows the transient ionization spectrum of $\mathrm{Na}_{3}$ using $60 \mathrm{fs}$ pulses at $623 \mathrm{~nm}$. At delay time $t=0$, when pump and probe pulses overlap, the total intensity is high enough to induce fragmentation of the formed $\mathrm{Na}_{3}^{+}$clusters which leads to the observed dip in the $\mathrm{Na}_{3}^{+}$signal around zero delay time. This dip vanishes for lower laser intensities. In order to examine the induced dynamics we analyzed the fast Fourier transform (FFT) (fig. $2 b$ ) of the transient ionization spectrum.

The contributions in the FFT spectrum near 140 ,
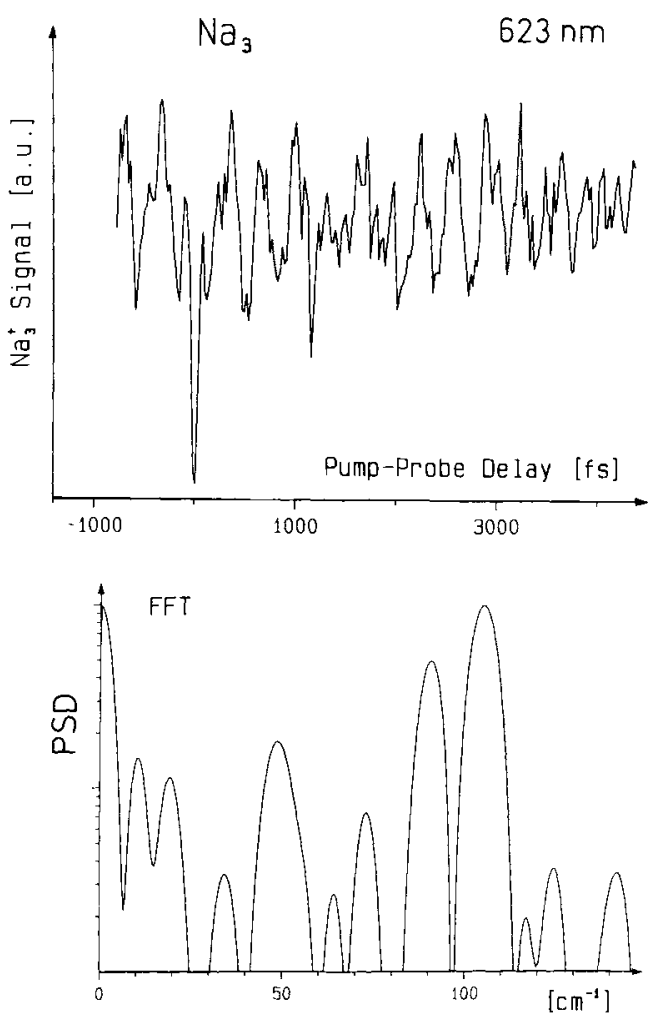

Fig. 2. Upper part: pump-probe ionization spectrum of the B state of the $\mathrm{Na}_{3}$ cluster using $60 \mathrm{fs}$ light pulses at $623 \mathrm{~nm}$. Lower part: Fourier (FFT) spectrum of the transient cluster $\left(\mathrm{Na}_{3}\right)$ ionization spectrum displayed in the upper part. 
90 and $50 \mathrm{~cm}^{-1}$ emerge only for high laser pulse intensities $\left(10^{10} \mathrm{~W} / \mathrm{cm}^{2}\right)$. We assign these frequencies on the basis of the spectroscopic analysis of Broyer et al. [20] to the symmetric stretch, to the asymmetric stretch and to the bending mode in the $\mathrm{Na}_{3}(\mathrm{X})$ electronic ground state. The observation of these frequencies clearly shows that at the applied laser intensities a ground state wavepacket is generated. Vibrational wavepacket motion in the electronic ground state of the dimer $\mathrm{Na}_{2}$ has also been reported recently [21]. Within the 60 fs pulse duration, the pump pulse with its spectral width of about $250 \mathrm{~cm}^{-1}$ coherently excites a number of levels and forms a wavepacket in the $B$ state and by stimulated emission pumping also in the $\mathrm{X}$ state. The three-dimensional vibrational wavepacket motion in the electronic ground state is monitored by ionizing $\mathrm{Na}_{3}(\mathrm{X})$ with two photons from the time-delayed probe laser pulse. Such a wavepacket motion can only be observed in the ion signal, when generation and ionization of the wavepacket take place in localized nuclear coordinate regions.

Besides the ground state dynamics, which is only induced at high laser intensities, there are other contributions in the FFT spectrum such as the dominant peak at $105 \mathrm{~cm}^{-1}$. With a pump pulse of a spectral bandwidth of $250 \mathrm{~cm}^{-1}$ and a central wavelength of $623 \mathrm{~nm}$ we can excite the $4{ }^{2} \mathrm{~A}_{1}$ and the $3{ }^{2} \mathrm{~B}_{2}$ state. According to the calculations of Meyer et al. [22] and Cocchini et al. [9] the symmetric stretch modes of these two states lie in the range of 94 to $111 \mathrm{~cm}^{-1}$. Thus a vibrational wavepacket motion in either one or both states may be responsible for the observed frequencies around $105 \mathrm{~cm}^{-1}$. The contribution around $74 \mathrm{~cm}^{-1}$ is tentatively assigned to the bending and asymmetric stretch mode of the $3{ }^{2} \mathrm{~B}_{2}$ state.

The other observed frequencies $8.5-12,17.5-20$ and $30.5-35 \mathrm{~cm}$ are assigned to a free pseudorotational wavepacket motion in the potential surface of the B state. The Jahn-Teller-deformed potential surface is described in the coordinate frame of the bending and the asymmetric stretch mode, while the totally symmetric stretch mode is not affected by the Jahn-Teller effect. Delacretaz et al. [11] interpreted their high-resolution two-photon ionization spectrum of the B-X transition in the framework of a pure Jahn-Teller model. The vibrational motion in the Jahn-Teller-distorted potential surface can be sep- arated into two independent motions, a radial vibrational motion which is characterized by the quantum number $v$, and an (angular) pseudo-"rotational" motion around the Jahn-Teller-affected potential surface, which resembles a mexican hat with three bowles. In the framework of a pure Jahn-Teller model the pseudorotation levels are described by half-integer $j$ quantum numbers. From the observed frequencies in the FFT of the transient ionization spectrum we conclude that a free pseudorotation wavepacket motion in the $\mathrm{Na}_{3} \mathrm{~B}$ state occurs. The contribution at $8.5-12 \mathrm{~cm}^{-1}$ is assigned to a wavepacket motion resulting from the coherent superposition of the levels $v=0,1 ; j=-3 / 2, j^{\prime}=3 / 2$. The other observed frequencies, $17.5-20$ and $30.5-35$ $\mathrm{cm}^{-1}$ are also assigned according to the analysis of Delacretaz et al. [11] and are due to the coherent superposition of the levels $v=0,1 ; j=3 / 2,5 / 2$ and $v=0,1 ; j=7 / 2,9 / 2$. In contrast to the high-resolution experiment of Delacretaz et al. [11] we find in the $(623 \mathrm{~nm}) \mathrm{Na}_{3}^{+}$FFT spectrum only a very minor contribution of the corresponding radial component $\left(\omega=128 \mathrm{~cm}^{-1}\right)$. This is probably due to the employed central wavelength of the pump pulse $(623$ $\mathrm{nm}$ ), which barely couples the radial vibronic levels $v=0$ and $v=1$. As already mentioned, Meiswinkel and Köppel [12] showed that the experiment of Delacretaz et al. could equally well be understood in the framework of a pseudo-Jahn-Teller model. In that case the accidental degeneracy of the $D_{3 \mathrm{~h}}$ states $3^{2} \mathrm{E}^{\prime}$ and $2{ }^{2} \mathrm{~A}_{1}^{\prime}$ is removed by vibronic coupling to the asymmetric vibrational $E^{\prime}$ mode. Within this model the pseudorotational levels are assigned to integer $j$ values. On the basis of the pseudo-Jahn-Teller model we assign the observed radial vibrational frequency of $\omega \approx 128 \mathrm{~cm}^{-1}$ to the degenerate bending and asymmetric modes in the $4^{2} A_{1}$ state. From our measurements however, we cannot decide which of these two models is more appropriate to describe the observed dynamics, because our method is only sensitive to pseudorotational energy differences.

In an additional real-time experiment we measured the two-photon ionization induced ZEKE photoelectrons as a function of the pump-probe delay time (fig. 3a), using 60 fs light pulses at a wavelength of $618 \mathrm{~nm}$. ZEKE photoelectron spectroscopy is only sensitive to very slow electrons, which are formed just in the vicinity of the ionization thresh- 

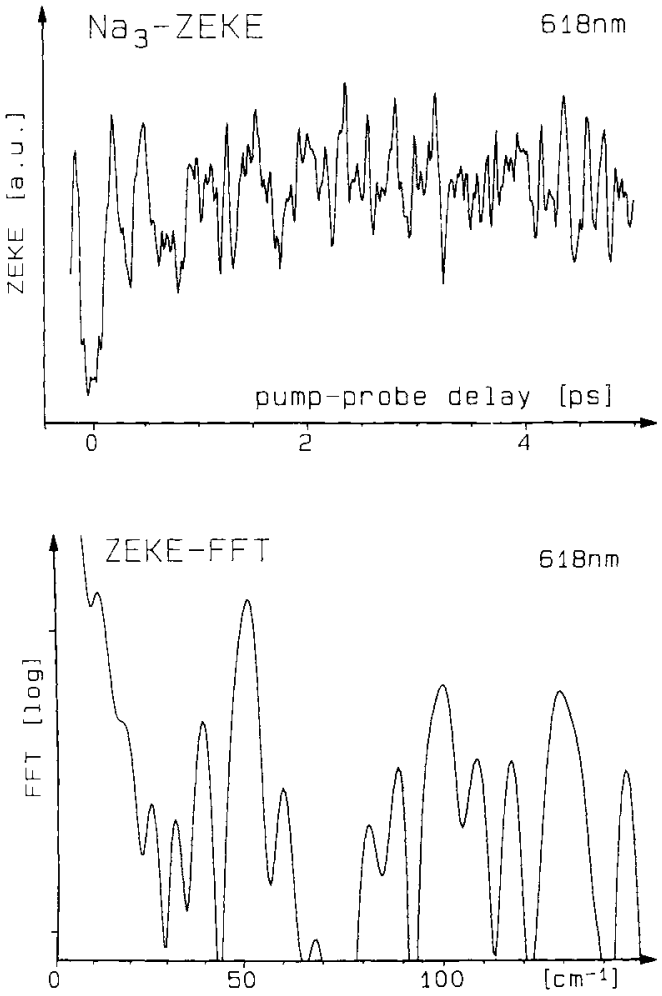

Fig. 3. Upper part: transient ZEKE photoelectron spectrum from femtosecond pump-probe ionization of the $\mathrm{B}$ state of $\mathrm{Na}_{3}$ with $618 \mathrm{~nm}$ photons. Lower part: Fourier (FFT) spectrum of the transient $\mathrm{Na}_{3}$ ZEKE photoelectron spectrum displayed in the upper part.

olds. It is well established now [2] that even for small metal clusters such as $\mathrm{Na}_{3}$ and $\mathrm{Na}_{4}$, the ZEKE signal is mainly produced by field ionization at highly excited Rydberg states. In this respect it was somewhat surprising to find a ZEKE FFT spectrum (fig. $3 b$ ) very similar compared with the FFT of the transient $\mathrm{Na}_{3}^{+}$spectrum (fig. 2b). The actually measured transient ZEKE (ionization) spectrum in fig. 3a looks as complex as the transient $\mathrm{Na}_{3}^{+}$spectrum in fig. 2a. It also shows the dip around $t=0$. Furthermore the frequencies supporting the vibrational wavepacket motion in the $\mathrm{X}$ and in the $\mathrm{B}$ state and the pseudorotational wavepacket motion in the $B$ state are again clearly observed. The individual amplitudes are somewhat different. In particular the contribution at $90 \mathrm{~cm}^{-1}$ is much weaker in the ZEKE FFT spectrum, and the peak around $128 \mathrm{~cm}^{-1}$, probably due to the $\mathrm{B}$ state radial vibrational motion, is clearly seen now in contrast to the $\mathrm{Na}_{3}^{+} \mathrm{FFT}$ spectrum. These differences are probably due to the different photon energy of 618 versus $623 \mathrm{~nm}$ and the different ionization processes. A pump laser at $618 \mathrm{~nm}$ with its $250 \mathrm{~cm}^{-1}$ spectral bandwidth can coherently couple the radial $v=0, v=1$ and $v=2$ vibronic levels, thus forming an observable radial vibrational wavepacket.

However, both independent experiments, the $\mathrm{Na}_{3}^{+}$and the ZEKE transient spectra, clearly show, that on a time scale of up to 10 ps we do not observe a fragmentation of the intermediate $B$ state. This result is in agreement with earlier nanosecond lifetime measurements [14]. The recently reported fast decay ( $6 \mathrm{ps} / 3-4 \mathrm{ps}$ ) of the B state by Wöste and coworkers $[15,16]$ is in clear disagreement with our measurements. In this respect it is not obvious to us how nanosecond and cw high-resolution two-photon ionization measurements can be performed with such a fast decaying resonant intermediate state. The proposed [16] coupling to so-called "dark states" which should have zero transition probability for the ionization process can in our opinion not account for the observed fast decay, because our transient ionization signals clearly show the vibrational $(105,74$, $128 \mathrm{~cm}^{-1}$ ) and pseudorotational motion in the $B$ state. Maybe this discrepancy is due to the employed laser intensities or the different coherences induced by light pulses with 20 times different pulse durations. Further experiments with varying pulse durations are in progress.

\section{Conclusion}

Femtosecond time-resolved spectroscopy has been applied to study the two-photon ionization dynamics of $\mathrm{Na}_{3}$ via the resonant $\mathrm{B}$ state. We observed threedimensional wavepacket motions in both the excited $\mathrm{B}$, and due to stimulated emission pumping during the pump pulse, also in the $\mathrm{X}$ electronic ground states. In the $B$ state we observed a free pseudorotation wavepacket motion in the Jahn-Teller-distorted potential surface. On time scales of up to $10 \mathrm{ps}$ we did not find a fragmentation of the $B$ state. 


\section{Acknowledgement}

This work has been supported by the Deutsche Forschungsgemeinschaft through the Sonderforschungsbereich 276. The long term loan of an $\mathrm{Ar}^{+}$ laser from Coherent Inc. is gratefully acknowledged.

\section{References}

[1] Proceedings of the Vth International Meeting on Small Particles and Inorganic Clusters, Z. Physik D 19/20 (1991); Proceedings of the VIth International Meeting on Small Particles and Inorganic Clusters, Z. Physik D (1993), in press.

[2] R. Thalweiser, S. Vogler and G. Gerber, Phys. Rev. Letters, submitted for publication; SPIE Proceedings Vol. 1858-22 (1993), in press.

[3] B. Bühler, R. Thalweiser and G. Gerber, Chem. Phys. Letters 188 (1992) 247.

[4] M. Broyer, G. Delacretaz, P. Labastie, R.L. Whetten, J.P. Wolf and L. Wöste, Z. Physik D 3 (1986) 131.

[5] K. Selby, M. Vollmer, J. Masui, V. Kresin, W.A. de Heer and W.D. Knight, Phys. Rev. B 40 (1989) 5417; C.R. Wang, S. Pollak, D. Cameron and M.M. Kappes, J. Chem. Phys. 93 (1990) 3787.

[6] C. Rothenfusser, C. Röttgermann, R. Thalweiser, V. Weiss and $\mathrm{G}$. Gerber, to be published.

[7] C. Rothenfusser, R. Thalweiser and V. Weiss, to be published.

[8] Y. Wang, T.F. George, D.M. Lindsay, A.C. Beri and L. Chu, J. Chem. Phys. 86 (1987) 3493;

F. Spiegelmann and D. Pavolini, J. Chem. Phys. 89 (1988) 4954;
V. Bonancic-Koutecky, I. Boustani, M. Guest and J. Koutecky, J. Chem. Phys. 89 (1988) 4861.

[9] F. Cocchini, T.H. Upton and W. Andreoni, J. Chem. Phys. 88 (1988) 6068.

[10] A. Herrmann, M. Hofmann, S. Leutwyler, E. Schumacher and L. Wöste, Chem. Phys. Letters 62 (1979) 216.

[ 11 ] G. Delacretaz, E.R. Grant, R.L. Whetten, L. Wöste and J.W. Zwanziger, Phys. Rev. Letters 56 (1986) 2598.

[12] R. Meiswinkel and H. Köppel, Chem. Phys. 144 (1990) 117.

[13] W.E. Ernst and S. Rakowsky, Z. Physik D 26 (1993), in press;

S. Rakowsky, F.W. Herrmann and W.E. Ernst, Z. Physik D 26 (1993), in press.

[14] M. Broyer, G. Delacretaz, Ni Guoquan, J.P. Wolf and L. Wöste, Chem. Phys. Letters 145 (1988) 232.

[15] E. Schreiber, H. Kühling, K. Kobe, S. Rutz and L. Wöste, Ber. Bunsenges. Physik. Chem. 96 (1992) 1301.

[16] S. Rutz, K. Kobe, H. Kühling, E. Schreiber and L. Wöste, Z. Physik D 26 (1993), in press.

[17] L. Khundkar and A.H. Zewail, Ann. Rev. Phys. Chem. 41 (1990) 15, and references therein.

[18] T. Baumert, B. Bühler, R. Thalweiser and G. Gerber, Phys. Rev. Letters 67 (1991) 3573;

T. Baumert, B. Bühler, M. Grosser, R. Thalweiser, V. Weiss, E. Wiedenmann and G. Gerber, J. Phys. Chem. 95 (1991) 8103;

M. Dantus, M.H.M. Janssen and A.H. Zewail, Chem. Phys. Letters 181 ( 1991 ) 281 .

[19] K. Müller-Dethlefs, M. Sander and E.W. Schlag, Z. Naturforsch. 39a (1984) 1089.

[20] M. Broyer, G. Delacretaz, G.Q. Ni, R.L. Whetten, J.P. Wolf and L. Wöste, Phys. Rev. Letters 62 (1989) 2100.

[21] T. Baumert, V. Engel, C. Meier and G. Gerber, Chem. Phys. Letters 200 ( 1992 ) 488.

[22] W. Meyer, private communication. 